\title{
Estequiometría y ley de conservación de la masa: lo que puede ocultar la simplificación del discurso experto
}

\author{
Stoichiometry and the law of mass conservation: what can be \\ hidden behind the simplification of the experts' discourse
}

 \\ Salvador $\mathrm{Ali}^{3}$
}

\begin{abstract}
Resumen: En el presente trabajo se analiza el tema estequiometría, en su relación con la Ley de Conservación de la Masa, desde la perspectiva de análisis comunicacional en el aula de química para la interpretación de errores de los estudiantes. La revisión del discurso docente a través de libros de textos permite reflexionar sobre los significados otorgados por los docentes a los términos "reacción química", "reactivos" y "productos", y sobre qué podrían comprender los estudiantes, cuando se omiten reflexiones históricas y epistemológicas sobre dichos términos.
\end{abstract}

Palabras clave: Estequiometría. Conservación de la masa. Enseñanza de la Química. Lenguajes químicos. Concepciones alternativas.

\begin{abstract}
A deep perspective on students' misconceptions on stoichiometry and Mass Conservation Law is presented. An evaluation of chemical discourse through textbooks allows teachers to be aware of the meaning of "chemical reaction", "reagents" and "products" when talking with students. In addition, an analysis of how the omission of historical and philosophical reflections may lead to students' misconceptions is also presented.
\end{abstract}

Keywords: Stoichiometry. Mass conservation. Chemistry teaching. Chemical languages. Misconceptions.

\footnotetext{
${ }^{1}$ Centro de Formación e Investigación en Enseñanza de las Ciencias (CEFIEC), Grupo de Investigación en Aprendizaje y Didáctica de las Ciencias Naturales (GIADiCIeN), Facultad de Ciencias Exactas y Naturales. Universidad de Buenos Aires (UBA), Ciudad Universitaria, Pabellón II, 1428, Buenos Aires, Argentina. Email: $<$ lydia.galagovsky@gmail.com>

${ }^{2,3}$ Departamento de Ciencias Naturales, Facultad de Ciencias Exactas y Naturales, Universidad de Buenos Aires (UBA), Buenos Aires, Argentina.
} 


\section{Introducción}

Numerosas investigaciones dan cuenta de las dificultades de aprendizaje de la química por los estudiantes novatos (BARKER, 2000; BEN-ZVI; GAI, 1994; CARMICHAEL et al., 1990; GILBERT; TREAGUST, 2009; JOHNSTONE, 1991, 2000, 2010; McCLARY, TALANQUER, 2011a, 2011b; MAEYER; TALANQUER, 2010; TABER, 2001, 2002, 2009; TALANQUER 2009, 2010a, 2010b, 2011). Carr (1984) había argumentado que muchos problemas de aprendizaje de Química pueden catalogarse como de "confusión de modelos" debiéndose a que la disciplina cuenta generalmente con varios modelos para un mismo tópico (GROSSLIGHT et al., 1991). De Vos, Bulte y Pilot (2003) señalan que el currículo de química es de tipo sedimentario, ubicando capa sobre capa contenidos científicos que se fueron revelando en épocas históricas y desde modelos diferentes.

Evidentemente, la química es una disciplina científica cuyos conceptos fundamentales (elemento, sustancia, molécula, ion, interacciones, etc.) son abstractos y sus modelos teóricos han evolucionado históricamente de forma no lineal. En el trabajo docente, se recortan los contenidos a enseñar y se secuencian desde tradiciones escolares con la mejor intención de elegir modelos apropiadamente simplificados para que sean accesibles a los estudiantes. Sin embargo, dado que el discurso químico no resulta fácil de comprender para los estudiantes novatos, se propone en este trabajo analizar la clase de química como un espacio de comunicación entre el docente experto en ciertas temáticas y sus estudiantes. Al aplicar el paradigma de la comunicación (SHANNON; WEAVER, 1948) al aula de química, se considera que el mensaje a transmitir en el intercambio comunicacional es un discurso erudito, con terminología y explicaciones científicas, generado por el docente o expresado en textos específicos.

Decodificar un mensaje es interpretarlo desde la significación que le ha otorgado el emisor. Así, el estudiante receptor del discurso químico debe comprender el tema, y resolver problemas aplicando ese conocimiento con manejo apropiado de los lenguajes y modelos científicos subyacentes. Se espera que el estudiante, además de "hablar ciencia" (LEMKE, 1997) pueda comprenderla.

En trabajos previos de los autores se ha discutido cómo elementos presentes en el mensaje (discurso erudito) pueden ser origen de obstáculo.s para el aprendizaje (GALAGOVSKY; DI GIACOMO; CASTELO, 2009; GALAGOVSKY et al., 2014). Desde esta perspectiva de la comunicación en el aula, es necesario el análisis de la complejidad del propio discurso químico experto y de sus implicancias. Esto debe ser estudiado tanto sobre el procesamiento cognitivo de estudiantes novatos, como desde la escasa discriminación conceptual oportuna cuando los expertos realizan transposiciones didácticas irreflexivas. En el presente trabajo se continúa, desde dicha perspectiva, la interpretación de errores de los estudiantes sobre el tema estequiometría en relación con la Ley de Conservación de la Masa (GALAGOVSKY; GIUDICE, 2015).

\section{Las respuestas de alumnos dan el alerta}

El tema estequiometría está incluido en la enseñanza y en las evaluaciones de la primera asignatura de química en la Universidad de Buenos Aires (Argentina). De las respuestas de algunos estudiantes en una de esas evaluaciones, surgieron evidencias específicas sobre obstáculos en la 
discriminación del concepto de conservación de la masa, dentro del tema estequiometría. Esto condujo a un trabajo de investigación sobre problemas de aprendizaje acerca de las reacciones químicas (DI GIACOMO; ALÍ, 2012) y a plantear ciertas reflexiones sobre la enseñanza del tema (GALAGOVSKY et al., 2014). Se presenta a continuación el ejemplo que nos permitirá profundizar en esas reflexiones.

En un recipiente cerrado que contiene metano y oxígeno gaseosos ocurre la reacción química representada por la siguiente ecuación: $\mathrm{CH}_{4}(\mathrm{~g})+2 \mathrm{O}_{2}(\mathrm{~g}) \rightarrow \mathrm{CO}_{2}(\mathrm{~g})+2 \mathrm{H}_{2} \mathrm{O}(\mathrm{g})$ a) Se colocan $\mathbf{8} \mathbf{g}$ de metano y $\mathbf{4 0} \mathbf{g}$ de oxígeno y se espera que la reacción finalice. ¿Qué sustancias estarán presentes en el sistema final y cuál será la masa de cada una de ellas?

b) Se realiza la reacción anterior con la misma masa de metano pero con $\mathbf{4 5} \mathbf{g}$ de oxígeno. Indique si la siguiente afirmación es correcta o incorrecta, explicando con palabras su decisión: «La suma de las masas de dióxido de carbono y agua será $\mathbf{5 3} \mathbf{g}$ por la ley de la conservación de la masa».

Se esperaba que en la pregunta (a) los estudiantes contestaran que el sistema final tendría una masa de $48 \mathrm{~g}$ - igual que la inicial - donde habría $8 \mathrm{~g}$ de $\mathbf{O}_{2}$ sin reaccionar, $22 \mathrm{~g}$ de $\mathbf{C O}_{2}$ y $\mathbf{1 8} \mathbf{g}$ de $\mathbf{H}_{2} \mathbf{O}$. Para la pregunta (b) se esperaba que se reconociera una masa del sistema final igual a la inicial de $\mathbf{5 3} \mathbf{g}$, por la ley de conservación de la masa, distinguiendo que en el sistema final la masa de los productos $\left(\mathrm{CO}_{2} \mathrm{y} \mathrm{H}_{2} \mathrm{O}\right)$ no se modificaría, pero habría también 13 g de oxígeno sin reaccionar.

Efectivamente, se encontraron numerosas respuestas erróneas. Un ejemplo típico fue la consideración de las masas de dióxido de carbono y agua como sinónimo de "sistema final", sin considerar en él el exceso de oxígeno. Este tipo de razonamiento fue generalmente confirmado en las respuestas a la pregunta (b), con argumentos tales como: "No se conserva la masa porque la suma de $\mathrm{CO}_{2}$ y $\mathrm{H}_{2} \mathrm{O}$ da $40 \mathrm{~g}$ ". Es decir, los estudiantes confirman que el aumento de la masa del reactivo en exceso no provoca un aumento en la masa de los productos obtenidos $\left(\mathrm{CO}_{2} \mathrm{y}\right.$ $\left.\mathrm{H}_{2} \mathrm{O}\right)$. Sin embargo, resuelven el conflicto que les genera la diferencia entre la masa total inicial y final negando el cumplimiento de la ley de conservación de la masa.

Este tipo de respuestas en los estudiantes con inferencias erradas desde el punto de vista científico generó un alerta respecto de una significación solapada entre la macroscópica ley de conservación de la masa y el concepto de conservación del número de átomos - y de sus masas - entre el sector de reactivos y productos de una ecuación química balanceada. Esta reflexión continúa la visión ya planteada en el trabajo de Galagovsky y Giudice (2015). Cabía cuestionarse, entonces, si esta confusión entre modelos macroscópicos y submicroscópicos (TALANQUER, 2011, 2012) era sólo un problema local, o si se encontraba algún referente en la bibliografía que diera cuenta de una posible generalización del mismo. 


\section{$¿$ Que dicen los libros de texto sobre la Ley de Conservación de la Masa y su relación con la estequiometría?}

La idea de la conservación de la materia ya estaba presente en los filósofos presocráticos. Sin embargo, fue a partir de la experimentación cuantitativamente meticulosa, y de la concepción de sistema cerrado por parte de Lavoisier (BOIDO, 1989; GHINI; SANTOS, 2011; HUDSON, 1993) que pudo enunciarse la Ley de Conservación de la Masa (a mediados de la década de 1770), y así comenzó el despegue de la alquimia hacia la moderna ciencia química. Dalton, por su parte, atribuyó a los átomos - inmutables - de cada elemento un peso característico, con lo cual se dio fundamento a la idea de conservación de los mismos en una reacción química. Arribar a los acuerdos básicos del lenguaje de ecuaciones químicas balanceadas para aplicar a la estequiometría fue un largo proceso posterior de al menos un siglo.

La ley de conservación de la masa y la estequiometría están presentes en todos los libros de Química General, aunque en diferentes capítulos. La enseñanza de la ley de conservación de la masa es una de las primeras acciones tanto de un curso de química - de cualquier nivel como de los textos de química. En cambio, la enseñanza de estequiometría suele presentarse en clases posteriores, luego del tratamiento de otros temas tales como sistemas materiales, tabla periódica, uniones químicas. Generalmente, los docentes al presentar el tema de ecuaciones químicas y su balance mencionan la conservación de la masa. Sin embargo, no se aclara que este balance de masa a nivel atómico molecular no se corresponde unívocamente a la histórica Ley de Conservación de la Masa enunciada por Lavoisier.

¿Es similar el tratamiento de estos temas en los libros de texto? Hemos comprobado este solapamiento conceptual con diversas variantes. Por ejemplo, libros utilizados en la parte inicial de la segunda mitad del siglo XX daban cuenta de parte de ese proceso histórico de la ley de conservación de la masa. Por ejemplo, el libro de Sienko y Plane (1961) - desde una redacción consustanciada con la visión positivista de la ciencia - en el inicio de su capítulo 2 hace una breve referencia histórica a experimentos de Lavoisier y también de Landolt (a final del siglo XIX) que permitieron confirmar que la masa no se crea ni se destruye y que no se verifica la conversión de masa en energía durante las reacciones químicas. Posteriormente, en el capítulo 5 sobre estequiometría señala simplemente que: "La estequiometría se ocupa de cambios en los pesos en las reacciones químicas." (SIENKO; PLANE, 1961, p. 20, nuestra traducción).

En otro ejemplo, en el libro de Berry, Rice y Ross (1980) de Físico Química avanzada no se hace mención a Lavoisier y, desde una posición positivista de las ciencias expresa en la primera página del primer capítulo:

[...] Su objetivo [de la Química] era la construcción de teorías para correlacionar y predecir las regularidades. La más importante de estas regularidades fue la ley de Conservación de la Masa que enuncia que la masa total $[\ldots]$ presente es la misma antes y después de una reacción química. Una vez que esta ley fue reconocida uno puede rastrear las cantidades de varias clases de materia involucradas en las reacciones, y así determinar la composición de sustancias. (BERRY; RICE; ROSS, 1980 , p. 3, nuestra traducción) 
En textos de edición más reciente se tiende a presentar la aplicación de la ley de conservación de la masa como herramienta algorítmica para el balanceo de las ecuaciones químicas. Se mencionan a continuación tres ejemplos:

i. En el libro de química universitaria de nivel introductorio de Atkins y Jones (2006), si bien en ningún momento se menciona a Lavoisier - no figura ni en el índice temático -, en la sección inicial de Fundamentos, se hace referencia a la ley de conservación de la masa con la conexión directa a la explicación de cómo se procede a balancear una ecuación química. El texto dice:

[...] cuando se lleva a cabo una reacción en un recipiente cerrado, entonces la masa no cambia. La observación de que la masa total es constante durante una reacción química, recibe el nombre de ley de conservación de la masa. Dado que los átomos no se crean ni se destruyen, los químicos consideran cada símbolo elemental como la representación de un átomo del elemento, y luego multiplican las fórmulas por los factores adecuados para mostrar el mismo número de átomos de cada elemento a cada lado de la flecha. Se dice que la expresión resultante está equilibrada y se la llama ecuación química. (ATKINS; JONES, 2006, p. F57-F58)

ii. Di Risio, Roverano y Vazquez (2006, p. 353) presentan la ley de la conservación de la masa y mencionan a Lavoisier en las primeras páginas del libro, para retomar la idea en el capítulo 10 (Los Cambios Químicos) con el mismo fin señalado en el ejemplo anterior, diciendo: "Las ecuaciones químicas deben cumplir con la ley de conservación de la masa [Ley de Lavoisier]. Es decir, la masa total de las sustancias reaccionantes debe ser igual que la masa total de los productos de la reacción.”

iii. Por su parte, Ferrari et al. (2007, p. 60) presentan el siguiente enunciado simplificando y solapando la significación macroscópica de la ley con su aplicación a estequiometría: "[...] en todos los cambios o reacciones químicas la cantidad de materia inicial la de los reactantes] es igual a la final [la de los productos], en otras palabras, permanece constante."

En enunciados como los ejemplificados en estos libros no se distingue claramente que una reacción química debe analizarse desde la situación macroscópica (debe visualizarse el mismo recipiente antes y después de la reacción) y la representación simbólica de su ecuación química correspondiente, para la cual se requiere la interpretación submicroscópica - de nivel atómico-molecular - de su proceso, con códigos consensuados y específicos del leguaje de fórmulas.

\section{Qué oculta la simplificación}

Talanquer y Pollard (2010) señalan que los currículos de las asignaturas introductorias de química suelen estar llenos de definiciones y procesos algorítmicos de resolución de problemas con los que se puede enfrentar un químico experto, pero pocas veces se pone énfasis 
en la forma en que los químicos piensan. En el caso particular de este trabajo se muestra que, desde la enseñanza del tema estequiometría, debería poder cumplirse esta recomendación. Un adecuado tratamiento histórico y epistemológico permitiría superar muchas de las dicotomías con las que se convive en educación química, como las cuestiones abstracto versus concreto, macroscópico versus simbólico, algorítmico versus conceptual, etc. (TALANQUER, 2012).

En la red de la Figura 1 se resumen las ideas principales de este trabajo, que se articulan con las ya presentadas en el artículo antes mencionado (GALAGOVSKY; GIUDICE, 2015). Más allá de la división en capítulos o en clases que distancian al concepto histórico de Ley de Conservación de la Masa de los conceptos involucrados en estequiometría, es necesario desde la enseñanza reconsiderar el rol central del concepto de "reacción química", para llegar a él desde las interpretaciones de los niveles macroscópico y simbólico.

Figura 1. Una propuesta con reflexión histórico-epistemológica para el planteo del concepto de "reacción química" y su relación con la ley de conservación de la masa y los procedimientos algorítmicos de estequiometría

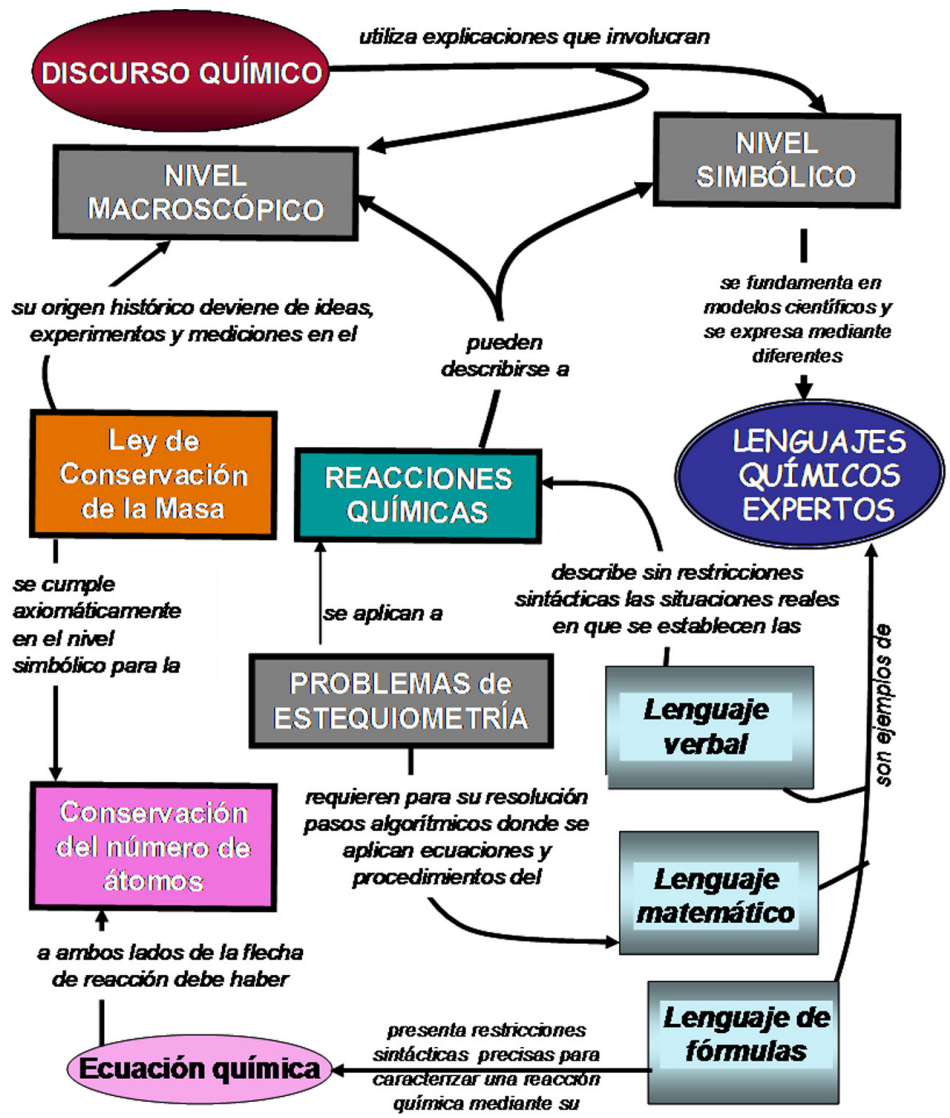

Fuente: Elaborado por los autores. 
La simplificación del relato que omite la reflexión histórica sobre la evolución del concepto de reacción química, no distingue la naturaleza epistemológicamente diferente entre la construcción del modelo propuesto por Lavoisier en el siglo XVIII y la aproximación estequiométrica cuyo consenso comenzó a finales del siglo XIX. Esta distinción requiere reconocer inicialmente que en una reacción química el modelo de cuantificación del peso sobre el sistema cerrado inicial y final es conceptualmente diferente al modelo estequiométrico de conservación del número de átomos en reactivos y productos. Este modelo estequiométrico deriva de avances científicos que requirieron décadas de discusiones hasta llegar al consenso sobre nomenclaturas y reglas sintácticas que subyacen al lenguaje de fórmulas y al conocimiento particular y específico de cada reacción química.

La pregunta necesaria para los docentes sería qué se quiere significar con los términos "reactivos" o "productos". Es decir, la forma de redacción de la ley de Conservación de la Masa y su relación con el concepto de estequiometría puede resultar correcta o no, según la interpretación que se haga de esos dos términos. El experto puede comprender las diferencias entre los términos "producto" y "estado final". Los estudiantes, en cambio, generalizan el término "productos" y lo analogan al de "sistema final". De esta manera podrían aplicar la Ley de Conservación de la Masa sin tener en cuenta ni la presencia de impurezas ni la de reactivos en exceso que queden sin reaccionar, como tampoco los casos de reacciones reversibles en los cuales el sistema final está constituido también por reactivos.

\section{El "hablar química" versus el "comprender química"}

El discurso de Química se expresa en muy diversos lenguajes para representar sus explicaciones abstractas: lenguaje verbal en explicaciones y textos; lenguaje gráfico en dibujos y esquemas, tanto de nivel macroscópico como de nivel atómico-molecular; lenguajes matemáticos en ecuaciones y lenguaje de fórmulas químicas en ecuaciones químicas, etc. (GALAGOVSKY, 2008). En el discurso docente y en los libros de texto se utiliza una multiplicidad simultánea de lenguajes explicativos con el fin de representar la complejidad de los conceptos teóricos expertos, que son interpretaciones modeladas sobre funcionamientos a nivel atómico-molecular (GALAGOVSKY; DI GIACOMO; CASTELO, 2009; GALAGOVSKY et al., 2014).

Evidentemente, comprender química supone que docentes y estudiantes lleguen a compartir los significados científicos del discurso químico. Sin embargo, los ejemplos presentados en este trabajo dan cuenta de lo complejo que puede resultar a estudiantes novatos construir aprendizajes sobre temas centrales de la química a partir de discursos que simplifican conceptos pero no ayudan a discriminar los modelos científicos subyacentes. De esta manera los estudiantes quedan expuestos a la construcción de generalizaciones erróneas.

Talanquer (2012) plantea que en la comunidad de investigadores en educación en ciencias hay tensión sobre cómo abordar la dicotomía entre las concepciones alternativas de los estudiantes y los modelos científicos. Marín Martínez y Cárdenas Salgado (2011, p. 37) plantean que dentro de la analogía "el estudiante como un científico" se da "excesiva confianza en creer que las respuestas del alumno representan su conocimiento", poniendo en duda una necesaria concepción de que sus teorías implícitas sean formaciones cognitivas fuertes e inamovibles. Desde el enfoque aquí presentado se plantea que los estudiantes construyen ideas incorrectas 
sobre estequiometría y su relación con la Ley de Conservación de la Masa a partir de generalizar enunciados particulares, pero el propio discurso docente no está exento de responsabilidad.

En este trabajo se ha mostrado que el origen de significaciones erróneas que construyen los estudiantes puede partir de un discurso docente que simplifica y omite cuestiones históricas.

Oliva Martínez (1999) en una reseña sobre los marcos teóricos que sustentan la posibilidad de lograr un cambio conceptual en estudiantes de ciencias, ha señalado que los actuales modelos vigentes tienen escasa capacidad para esclarecer cuáles son los procesos internos que se producirían en la mente de los estudiantes durante el cambio de una idea por otra. En el presente trabajo en complemento con Galagovsky y Giudice (2015) se presenta un nuevo marco teórico para interpretar los errores de estudiantes en el tema de estequiometría en relación con la ley de conservación de la masa. Esta propuesta brinda reflexiones epistemológicas que derivan en estrategias de enseñanza más convenientes para los estudiantes, en tanto se asume que los contextos históricos no deben ser simples listados de datos (fechas, nombres, hechos), sino marcos de referencia sobre los modelos científicos que actuaban como plataformas desde las cuales se producía el conocimiento particular.

\section{Referencias}

ATKINS, P.; JONES, L. Principios de química: los caminos del descubrimiento. Buenos Aires: Editorial Médica Panamericana, 2006.

BARKER, V. Beyond appearances: students' misconceptions about basic chemical ideas: a report prepared for the Royal Society of Chemistry. London: Royal Society of Chemistry, 2000.

BEN-ZVI, N.; GAI, R. Macro- and micro-chemical comprehension of real-world phenomena: classroom knowledge versus the experience of the senses. Journal of Chemical Education, Washington, v. 71, n. 9, p. 730-734, 1994.

BERRY, R. S.; RICE, S.; ROSS, J. Phisical chemistry. New York: Wiley, 1980.

BOIDO, G. Lavoisier: la madurez de la química. Ciencia Hoy, Buenos Aires, v. 1, n. 3 , p. $78-80,1989$.

CARMICHAEL, P. et al. Research on students' conceptions in science: a bibliography. [Leeds]: University of Leeds: Centre for Studies in Science and Mathematics Education, 1990.

CARR, M. Model confusion in chemistry. Research in Science Education, Dordrecht, v. 14, n. 1, p. 97-103, 1984.

DE VOS, A. W.; BULTE, A. M. W.; PILOT, A. Chemistry curricula for general education: analysis and elements of a design. In: GILBERT, K. J. et al. (Ed). Chemical education: towards research-based practice. Dordrecht: Kluwer, 2003. p. 101-124. 
DI GIACOMO, M. A.; ALÍ, S. Reacciones químicas: dificultades en su aprendizaje. In: JORNADAS DE INGRESO Y PERMANENCIA EN CARRERAS CIENTÍfICO-TECNOLÓGICAS, 3., 2012, San Juan. Actas... San Juan: Editorial Universidad Nacional de San Juan, 2012. p. 151.

DI RISIO, C.; ROVERANO, M.; VAZQUEZ, M. I. Química básica. Buenos Aires: Educando, 2006.

FERRARI, A. et al. Física y química: naturaleza corpuscular y carácter eléctrico de la materia. Magnetismo. Fuerzas. Buenos Aires: Santillana, 2007.

GALAGOVSKY, L. Enseñanza de las ciencias naturales: un desafío a nivel mundial. El caso particular de enseñanza de la química. Revista Proyecciones, Buenos Aires, v. 6, n. 2, p. 23-36, 2008.

GALAGOVSKY, L.; DI GIACOMO, M. A.; CASTELO, V. Modelos vs. dibujos: el caso de la enseñanza de fuerzas intermoleculares. Revista Electrónica de Enseñanza de las Ciencias, Vigo, v. 8, n. 1, p. 1-22, 2009. Disponible en: <http://www.saum.uvigo.es/reec/ volumenes/volumen8/ART1_Vol8_N1.pdf>. Acceso el: 11 ago. 2014.

GALAGOVSKY, L.; GIUDICE, J. Estequiometría y ley de conservación de la masa: una relación a analizar desde la perspectiva de los lenguajes químicos. Ciência \& Educação, Bauru, v. 21, n. 1, 2015.

GALAGOVSKY, L. et al. Algunas reflexiones sobre la distancia entre "hablar química" y “comprender química”. Ciência \& Educação, Bauru, v. 20, n. 4, p. 785-799, 2014. Disponible en: < http://dx.doi.org/10.1590/1516-73132014000400002 >. Acceso el: 20 feb. 2015.

GHINI, A.; LANTOS, C. Contribución del conocimiento químico a la supervivencia humana: los casos de Lavoisier y Pasteur. In: GALAGOVSKY, L. R. (Ed.). Química y civilización. Buenos Aires: Asociación Química Argentina, 2011. p. 21-34.

GILBERT, J. K.; TREAGUST, D. (Ed.). Multiple representations in chemical education. Dordrecht: Springer, 2009.

GROSSLIGHT, L. et al. Understanding models and their use in science: conceptions of middle and high school students and experts. Journal of Research in Science Teaching, Hoboken, v. 28, n. 9, p. 799-822, 1991.

HUDSON, J. The history of chemistry. New York: Chapman and Hall, 1993.

JOHNSTONE, A. H. Teaching of chemistry: logical or psychological? Chemical

Education: research and practice in Europe, Ioannina, v. 1, n. 1, p. 9-15, 2000.

Why is science difficult to learn?: things are seldom what they seem. Journal of

Computer Assisted Learning, Malden, v. 7, n. 2, p. 75-83. 1991.

. You cant't get there from here. Journal of Chemical Education, Washington, v. 87 , n. 1, p. 22-29, 2010.

LEMKE, J. L. Aprender a hablar ciencia: lenguaje, aprendizaje y valores. Barcelona: Paidós Ibérica, 1997. 
McCLARY, L.; TALANQUER, V. College chemistry students' mental models of acids and acid strength. Journal of Research in Science Teaching, Hoboken, v. 48, n. 4, p. 396-413, 2011a.

. Heuristic reasoning in chemistry: making decisions about acid strength.

International Journal of Science Education, Abingdon, v. 33, n. 10, p. 1433-1454, 2011b.

MAEYER, J.; TALANQUER, V. The role of heuristics in students thinking: ranking of chemical substances. Science Education, Hoboken, v. 94, n. 6, p. 963-984, 2010.

MARÍN MARTÍNEZ, N.; CÁRDENAS SALGADO, F. A. Valoración de los modelos más usados en la enseñanza de las ciencias basados en la analogía "el alumno como científico".

Enseñanza de las Ciencias, Barcelona, v. 29, n. 1, p. 35-46, 2011.

OLIVA MARTÍNEZ. J. M. Algunas reflexiones sobre las concepciones alternativas y el cambio conceptual. Enseñanza de las Ciencias, Barcelona, v. 14, n. 1, p. 87-92, 1999.

SHANNON, C. E.; WEAVER, W. The mathematical theory of communication. Urbana: University of Illinois Press, 1948.

SIENKO, M. J.; PLANE, A. Chemistry. NewYork: McGraw Hill, 1961.

TABER, K. S. Building the structural concepts of chemistry: some considerations from educational research. Chemistry Education: research and practice in Europe, Ioannina, v. 2, n. 2, p. 123-158, 2001.

Chemical misconceptions: prevention, diagnosis and cure. London: Royal Society of Chemistry, 2002. v. 1.

. Learning at the symbolic level. In: GILBERT, J. K.; TREAGUST, D. (Ed.).

Multiple representations in chemical education. Dordrecht: Springer, 2009. p. 75-105.

TALANQUER, V. Chemistry education: ten dichotomies we live by. Journal of Chemical Education, Washington, v. 89, n. 11, p. 1340-1344, 2012.

Exploring dominant types of explanations built by general chemistry students.

International Journal of Science Education, Abingdon, v. 32, n. 18, p. 2393-2412, 2010a.

. On cognitive constraints and learning progressions: the case of structure of matter.

International Journal of Science Education, Abingdon, v. 31, n. 15, p. 2123-2136, 2009.

El papel de las ideas previas en el aprendizaje de la química. Alambique: didáctica de las ciencias experimentales, Barcelona, n. 69, p. 35-41, 2011.

Pensamiento intuitivo en química: suposiciones implícitas y reglas heurísticas.

Enseñanza de las Ciencias, Barcelona, v. 28, n. 2, p. 165-174, 2010 b.

TALANQUER, V.; POLLARD, J. Let's teach how we think instead of what we know.

Chemistry Education Research and Practice, Ioannina, v. 11, n. 2, p. 74-83, 2010.

Artigo recebido em 02/03/13. Aceito em 07/01/14 